\title{
Multidimensional weak resolvents and spatial equivalence of normal operators
}

\author{
by \\ Michą Jasiczak (Poznań)
}

\begin{abstract}
The aim of this paper is to answer some questions concerning weak resolvents. Firstly, we investigate the domain of extension of weak resolvents $\Omega$ and find a formula linking $\Omega$ with the Taylor spectrum.

We also show that equality of weak resolvents of operator tuples $A$ and $B$ results in isomorphism of the algebras generated by these operators. Although this isomorphism need not be of the form

$$
X \mapsto U^{*} X U,
$$

where $U$ is an isometry, for normal operators it is always possible to find a "large" subspace on which unitary similarity holds. This observation is used to prove that the infinite inflation of the spatial isomorphism between algebras generated by inflations of $A$ and $B$, respectively, does have the form (1).

These facts are generalized to other not necessarily commuting operators. We deal mostly with the self-adjoint case.
\end{abstract}

1. Introduction. Let $A$ be an operator on a Banach space $X$ with the dual space denoted by $X^{\star}$. The paper [13] (cf. also [12]) started a systematic study of sets of weak resolvents, i.e. functions of the form $\zeta \mapsto G\left((\zeta-A)^{-1} f\right)$, where $f \in X$ and $G \in X^{\star}$. It is natural to expect, especially in light of results in local spectral theory, that the set of all functions of this form, denoted by $W(A)$, should contain some relevant information about the operator $A$.

It was proved that for cyclic operators acting on a finite-dimensional space $W(A)$ is a complete similarity invariant of $A$. The same characterization was obtained for normal operators having spectra of zero area. It was also shown that an operator is algebraic if and only if $W(A)$ consists only of meromorphic functions.

In fact, the study of weak resolvents had started earlier. In [11] it was proved that if each weak resolvent of an operator $A$ belongs to the Hardy space $H^{1}(\mathbb{D})$, then the spectral radius of $A$ is less than one. This was gener-

2000 Mathematics Subject Classification: 47B15, 47C05, 47L99.

Key words and phrases: weak resolvent, spatial isomorphism, Taylor spectrum. 
alized to tuples of operators and Taylor spectrum in [9]. It should be pointed out that it is still not clear for which contractions weak resolvent sets are contained in other Hardy spaces.

Let $n \geq 1$ be an integer and $\mathbb{B}$ be a unit ball in $\mathbb{C}^{n}$. We will denote by $\mathcal{H}$ a Hilbert space and by $\mathbf{B}(\mathcal{H})$ the algebra of all bounded operators on $\mathcal{H}$. In [15] operator-valued Cauchy and Poisson kernels were defined. Let $A \in \mathbf{B}(\mathcal{H})^{n}$ be a commuting $n$-tuple. Then the operator-valued Cauchy kernel is defined by the formula

$$
C(A, \zeta)=[1-\langle A, \zeta\rangle]^{-n}=\left[1-\left(A_{1} \bar{\zeta}_{1}+\cdots+A_{n} \bar{\zeta}_{n}\right)\right]^{-n} .
$$

The Poisson kernel is given by

$$
P(A, \zeta)=C(A, \zeta) \Delta_{A}^{n} C\left(A^{*}, \bar{\zeta}\right),
$$

where $\Delta_{A}^{n}=\sum_{\alpha \geq 0} k_{n, \alpha} A^{* \alpha} A^{\alpha}$ (for the definition of the numbers $k_{n, \alpha}$ see [15]). Throughout the paper we use standard multi-index notation.

Assume that the Taylor spectrum of $A$ is contained in the unit ball. For functions in the ball algebra $A(\mathbb{B})$ the following formula holds:

$$
f(A)=\int_{S} f(\zeta) K(A, \zeta) d \sigma(\zeta)
$$

where $\sigma$ is the unique rotation-invariant normalized positive Borel measure on $S=\partial \mathbb{B}$ and $K(A, \zeta)$ is the Cauchy or Poisson kernel. Accordingly, it is natural to investigate properties of functions of the form

$$
\phi(\zeta)=\langle K(A, \zeta) f, g\rangle, \quad f, g \in \mathcal{H},
$$

which will thereafter be called multidimensional weak resolvents or briefly weak resolvents of an $n$-tuple $A$ of operators. For the operator-valued Cauchy kernel we may and will consider weak resolvents for commuting $n$-tuples of operators acting on a Banach space $X$.

It is the aim of this paper to give, at least partial, answers to some problems posed in [8] and [13]. We will show that weak resolvents of $n$-tuples of commuting operators cannot be used to investigate the Taylor spectrum. We will prove a relation between the Taylor spectrum and the domain of extension of each weak resolvent $\Omega(A)$. This fact, generalizing results of [9], shows that there exist plenty of operators for which $\Omega(A)$ is the same but whose Taylor spectra differ significantly.

We also investigate consequences of inclusions of the form $W(A) \subset W(B)$ for normal commuting operators. We deal mostly with the self-adjoint case. This is again connected with questions posed in [13] and [8].

For operators $A_{1}, \ldots, A_{n} \in \mathbf{B}(\mathcal{H})$ we will denote by $\mathcal{P}(A)$ the image of the homomorphism

$$
\mathbb{C}\left[Z_{1}, \ldots, Z_{n}\right] \ni p \mapsto p(A) \in \mathbf{B}(\mathcal{H})
$$


It is an easy consequence of the above-mentioned theorem of F.-H. Vasilescu, in light of results in [13], that the homomorphism $\Phi: \mathcal{P}(A) \rightarrow \mathcal{P}(B)$ defined by $p(A) \mapsto p(B)$ is bounded (homomorphisms of this kind are called spatial). Thus, if $W(A)=W(B)$ (this condition will be referred to as the resolvent equality) then $\overline{\mathcal{P}(A)}$ and $\overline{\mathcal{P}(B)}$ are isomorphic as Banach algebras.

It is natural to ask about the form of this isomorphism. It is almost obvious that the operators $A$ and $B$ need not be unitarily equivalent. However, we shall prove that for commuting normal operators the resolvent equality results in equivalence of the operators on each maximal cyclic subspace. This condition turns out to be also necessary for the weak resolvent equality to hold.

Although a spatial isomorphism need not be of the form $X \mapsto U^{*} X U$, where $U$ is unitary or isometric on $\mathcal{H}$, the condition $W\left(A, A^{*}\right)=W\left(B, B^{*}\right)$ implies that $\Phi: p\left(A, A^{*}\right) \mapsto p\left(B, B^{*}\right)$ can be written in the form

$$
\Phi\left(p\left(A, A^{*}\right)\right)=V^{*} p\left(A^{(\infty)}, A^{(\infty) *}\right) V \iota,
$$

where $A^{(\infty)}$ denotes the infinite inflation of $A$ and $\iota$ is the inclusion of a Hilbert space $\mathcal{H}$ into the infinite orthogonal sum of copies of $\mathcal{H}$. Again, this fact is a necessary condition for the weak resolvent equality.

We generalize these theorems to other operators using elementary $C^{*}$ algebra techniques.

Acknowledgments. The author wishes to thank the referee for suggestions which helped the author not only to improve the style of the paper but also correct some mistakes.

2. Connection with Taylor spectrum. Let $A_{1}, \ldots, A_{n}$ be commuting operators acting on a Banach space $X$. Define

$$
c(A):=\left\{z \in \mathbb{C}^{n}: 1-\langle A, z\rangle \notin \operatorname{Inv} \mathbf{B}(X)\right\} .
$$

Definition 1. For $f \in \mathcal{X}$ and $g \in \mathcal{X}^{\star}$, the function $\phi=\phi_{f, g}: c(A)^{\mathrm{c}} \rightarrow \mathbb{C}$ $\left(U^{\mathrm{c}}\right.$ denotes the complement of the set $U$ ) defined by

$$
\phi\left(\zeta_{1}, \ldots, \zeta_{n}\right)=\langle C(A, \zeta) f, g\rangle
$$

is called a weak (Cauchy) resolvent of the operators $A_{1}, \ldots, A_{n}$. The set of all functions of this form will be denoted by $W_{C}(A)$, or $W(A)$ if no confusion can occur.

Observe that, a priori, a function of the form (4) is well defined on $c(A)^{\mathrm{c}}$. Obviously, it may happen that it can be extended, as an antiholomorphic function, to a larger domain. A trivial example consists in taking $f$ in a joint invariant subspace of $A$ and $g \in X^{*}$ annihilating $[\mathcal{P}(A) f]$ (if $S$ is a subset of a Banach space, $[S]$ stands for the closure of the span of $S$ ). Then $\phi(\zeta)=$ 
$\langle C(A, \zeta) f, g\rangle=0$ on $c(A)^{\mathrm{c}}$ and $\phi$ can be extended as an antiholomorphic function to the whole $\mathbb{C}^{n}$.

We will show that $c(A)^{\mathrm{c}}$ is the largest set, in the sense of inclusion, on which one can define every function from $W(A)$. From the spectral mapping property of the Taylor spectrum [5] it follows that

$$
\begin{aligned}
c(A) & =\left\{z \in \mathbb{C}^{n}: 1 \in \sigma(\langle A, z\rangle)\right\} \\
& =\left\{z \in \mathbb{C}^{n}: \exists \zeta \in \sigma_{\mathrm{T}}(A), 1=\zeta_{1} \bar{z}_{1}+\cdots+\zeta_{n} \bar{z}_{n}\right\} .
\end{aligned}
$$

Let $U$ be an open connected set (domain) in $\mathbb{C}^{n}$ with $U \cap c(A)^{\mathrm{c}} \neq \emptyset$ such that for each weak resolvent $f \in W(A)$, there exists an antiholomorphic function $F: U \rightarrow \mathbb{C}$ which extends $f$. We denote by $\Omega(A)$ the union of all domains $U$ satisfying the above-described condition. It follows that $c(A)^{\mathrm{c}} \subset$ $\Omega(A)$. We investigate the reverse inclusion.

Let us also define a local version of $c(A)$ denoted by $c(A, x)$ (see also [6] and [14]). We will say that $z \in \mathbb{C}^{n}$ does not belong to $c(A, x)$ if there exists an antiholomorphic function $F: U_{z} \rightarrow X$ defined on an open neighbourhood $U_{z}$ of $z$ such that

$$
(1-\langle A, \zeta\rangle)^{n} F(\zeta)=x
$$

for $\zeta \in U_{z}$ (in the terminology of [14] this function is A-associated to $x$ ). From the definition it follows that the set $c(A, x)^{\mathrm{c}}$ is open. We will need the following lemma:

Lemma 1. Let $x_{\alpha}$ be elements of a Banach space $X$. The domain of absolute convergence of the power series $\sum_{\alpha} x_{\alpha} z^{\alpha}$ is equal to the interior of the set

$$
\bigcap_{\xi \in X^{\star}} \bigcup_{k=1}^{\infty}\left\{z \in \mathbb{C}^{n}: \sup _{\alpha}\left|\xi\left(x_{\alpha}\right) z^{\alpha}\right| \leq k\right\} .
$$

Proof. This follows from the Banach-Steinhaus theorem and the Abel lemma (see for example [10]).

Proposition 2. If $z \in \Omega(A)$, then for each $x \in X$ there exists an open neighbourhood $\mathcal{V}$ of $z$ and an antiholomorphic function $G$ such that

$$
C(A, \zeta) G(\zeta)=x \quad \text { for } \zeta \in \mathcal{V} .
$$

In other words, $\Omega(A) \cap c(A, x)=\emptyset$ for every $x \in X$.

Proof. Let $x \in \mathcal{X}$. From the definition, for each $z \in \Omega(A)$ there exists a domain $U$ containing $z$ and an antiholomorphic extension of each weak resolvent to the domain $U$.

Choose open polydiscs $P_{1}=P\left(z_{1}, r_{1}\right), \ldots, P_{m}=P\left(z_{m}, r_{m}\right)$ with closures contained in $U$ with $z_{1} \in c(A)^{\mathrm{c}}, z_{i} \in P_{i-1}$ for $i=2, \ldots, m$ and $z \in P_{m}$. We may assume that the Taylor series at $z_{k}$ of each weak resolvent is convergent on $P_{k}$, for each $k=1, \ldots, m$. 
Since $z_{1} \in P_{1}$, it follows from Lemma 1 that the Taylor series of $C(A, \zeta) x$ at $z_{1}$ converges uniformly on compact subsets of $P_{1}$. The sum of this series, denoted by $F_{1}$, satisfies for $\zeta \in P_{1} \cap \Omega(A)$ the equation

$$
(1-\langle A, \zeta\rangle)^{n} F_{1}(\zeta)=x .
$$

Both $F_{1}$ and $(1-\langle A, \zeta\rangle)^{n}$ are antiholomorphic. Therefore, (6) must also be satisfied for $\zeta \in P_{1}$. Inductively, for each $k=1, \ldots, m$ there exists an antiholomorphic function $F_{k}: P_{k} \rightarrow \mathbb{C}$ satisfying the equation and whose Taylor extension is convergent, by Lemma 1 , on the whole $P_{k}$. This proves the lemma since $z$ belongs to $P_{m}$.

If $\lambda$ belongs to the boundary of the spectrum of an operator $A$, then $\lambda-A$ is not a surjection. This is a consequence of the fact that the boundary of the set of invertible elements of a Banach algebra consists of topological zero divisors. This argument also shows that for $\zeta \in \partial c(A)$ the operator $1-\langle A, \zeta\rangle$ is a topological zero divisor. Consequently, its range is a proper subspace of $X$. We will use this fact in the proof of the following lemma.

Lemma 3. The set $\{x \in \mathcal{X}: \partial c(A) \backslash c(A, x) \neq \emptyset\}$ is of the first Baire category.

Proof. We may assume that $c(A)^{\mathrm{c}} \neq \mathbb{C}^{n}$. Take a sequence $\lambda_{k}$ which is dense in $\partial c(A)$ and define $M_{k}=\left\{x \in \mathcal{X}: \lambda_{k} \notin c(A, x)\right\}$. It is easy to show that

$$
\{x \in X: \partial c(A) \backslash c(A, x) \neq \emptyset\}=\bigcup_{k=1}^{\infty} M_{k} .
$$

Thus, it is enough to prove that each $M_{k}$ is of the first category. Take $x \in M_{k}$; then there is an open neighbourhood $U$ of $\lambda_{k}$ and an antiholomorphic function $F: U \rightarrow X$ such that

$$
(1-\langle A, z\rangle)^{n} F(z)=x .
$$

Consequently, $x$ belongs to the range of the operator $1-\left\langle A, \lambda_{k}\right\rangle$. Since $\lambda_{k} \in$ $\partial c(A), R\left(1-\left\langle A, \lambda_{k}\right\rangle\right)$ is a proper subspace of $\mathcal{X}$ and by the open mapping theorem, it is of the first Baire category. In other words, $M_{k}$ is contained in a set of the first category. This yields the desired conclusion.

Theorem 4. $\Omega(A) \cap \partial c(A)=\emptyset$. Consequently, $\Omega(A)=c(A)^{\mathrm{c}}$.

Proof. Lemma 3 shows that there exists $x \in \mathcal{X}$ such that $\partial c(A) \subset c(A, x)$. On the other hand, from Proposition 2 we know that $\Omega(A) \subset c(A, x)^{\mathrm{c}}$ for each $x \in X$. Thus $\Omega(A) \cap \partial c(A)=\emptyset$.

From the definition it follows that $c(A)^{\mathrm{c}} \subset \Omega(A)$. It is easy to show that if there existed $z \in \Omega(A) \backslash c(A)^{\mathrm{c}}$, then we could find $\zeta \in \Omega(A) \cap \partial c(A)$.

REMARK 1. The previous theorem can be proved in a simpler way. Namely, one can take a direct route, applying the Banach-Steinhaus The- 
orem and mimicking the proof of Proposition 2, to construct an antiholomorphic function $F: \mathcal{V} \rightarrow \mathbf{B}(X), \mathcal{V}$ an open neighbourhood of $z \in \Omega(A)$, satisfying (5). It is the impression of the author that the proof presented above is more instructive.

REMARK 2. It is easy to observe that instead of the operator-valued Cauchy kernel one can take any other operator-valued holomorphic or antiholomorphic kernel, for instance the Bergman kernel. We shall not pursue this observation further. Instead, in the next section we introduce the concept of a spatial isomorphism of finitely generated subalgebras of $\mathbf{B}(\mathcal{H})$, whose dual preserves elementary functionals. This notion allows us to relieve the considerations from a particular choice of a kernel function.

REMARK 3. Assume now that $A_{1}, \ldots, A_{n}$ and $B_{1}, \ldots, B_{n}$ are tuples of commuting operators. We could have defined weak resolvents as germs on $c(A)^{\mathrm{c}}$ (which is open) of functions of the form (4).

From the previous theorem it would then follow that if $W_{C}\left(A_{1}\right)=$ $W_{C}\left(B_{1}\right)$, then $c\left(A_{1}\right)=c\left(B_{1}\right)$. It is obvious that 0 belongs to $c(A)^{\mathrm{c}}$ for each $A \in \mathbf{B}(\mathcal{H})$. Consequently, if $W_{C}\left(A_{1}\right)=W_{C}\left(B_{1}\right)$, then $\sigma\left(A_{1}\right) \backslash\{0\}=$ $\sigma\left(B_{1}\right) \backslash\{0\}$.

From the equality $W_{C}\left(A_{1}, \ldots, A_{n}\right)=W_{C}\left(B_{1}, \ldots, B_{n}\right)$ we can only infer that $c\left(A_{1}, \ldots, A_{n}\right)=c\left(B_{1}, \ldots, B_{n}\right)$. It is easy to show that there exist $n$ tuples $A$ and $B$ such that $c(A)=c(B)$, but $\sigma_{\mathrm{T}}(A) \neq \sigma_{\mathrm{T}}(B)$.

3. Weak resolvents of normal operators. Assume that $A_{1}, \ldots, A_{n}$ and $B_{1}, \ldots, B_{n}$ are commuting $n$-tuples of operators on a Banach space $X$. It was proved in [8] that the equality of the sets of one-dimensional weak resolvents implies that the algebras generated by these operators are isomorphic. The same holds true in the case of multidimensional weak resolvents.

Let $f$ be an element of a Banach space $X$ and $g \in X^{\star}$. The symbol $\mathcal{A}_{X \times X}^{\star}$ will stand for the set of all elementary functionals acting on a subspace $\mathcal{A} \subset \mathbf{B}(X)$. Recall that a functional is called elementary if it is of the form $X \mapsto\langle X f, g\rangle$. We will use the notation

$$
\mathcal{P}\left(A_{1}, \ldots, A_{n}\right) \cong \mathcal{P}\left(B_{1}, \ldots, B_{n}\right)
$$

if the map $\Phi$ sending $p\left(A_{1}, \ldots, A_{n}\right)$ to $p\left(B_{1}, \ldots, B_{n}\right)$ extends to a bounded algebra isomorphism. Equivalently, it can be said that $\mathcal{P}\left(A_{1}, \ldots, A_{n}\right)$ and $\mathcal{P}\left(B_{1}, \ldots, B_{n}\right)$ are spatially isomorphic. The fact that $\Phi$ is an isomorphism and the dual map

$$
\Phi^{\star}: \mathcal{P}\left(B_{1}, \ldots, B_{n}\right)^{\star} \rightarrow \mathcal{P}\left(A_{1}, \ldots, A_{n}\right)^{\star}
$$

maps $\mathcal{P}\left(B_{1}, \ldots, B_{n}\right)_{\mathfrak{x} \times x}^{\star}$ onto $\mathcal{P}\left(A_{1}, \ldots, A_{n}\right)_{\mathfrak{X} \times x}^{\star}$ will be denoted by

$$
\mathcal{P}\left(A_{1}, \ldots, A_{n}\right) \approx \mathcal{P}\left(B_{1}, \ldots, B_{n}\right) .
$$


Theorem 5. Assume that $\sigma_{\mathrm{T}}(A), \sigma_{\mathrm{T}}(B) \subset \mathbb{B}$. The equality $W_{C}(B)=$ $W_{C}(A)$ holds if and only if $\mathcal{P}(A) \approx \mathcal{P}(B)$.

Proof. First of all, observe that if $W(B) \subset W(A)$ then the mapping $\Phi: p(A) \mapsto p(B)$ is well defined. Define continuous bilinear operators $a, b$ : $X \times X^{\star} \rightarrow C(\overline{\mathbb{B}})$ by

$$
a(f, g)=\langle C(A, \zeta) f, g\rangle, \quad b(f, g)=\langle C(B, \zeta) f, g\rangle .
$$

From the assumption it follows that the range of $b$ is contained in the range of $a$. Consequently, from the Grabiner theorem (see [8]) we infer that there exists a constant $c$ such that for each $\xi \in C(\overline{\mathbb{B}})^{\star}$,

$$
\|\xi \circ b\| \leq c\|\xi \circ a\| .
$$

Fix a polynomial $p \in \mathbb{C}\left[Z_{1}, \ldots, Z_{n}\right]$ and let

$$
\xi_{p}(f)=\int_{S} f(z) p(z) d \sigma(z) \quad \text { for } f \in C(\overline{\mathbb{B}}) .
$$

Since by $(3), \xi_{p}(a(f, g))=\langle p(A) f, g\rangle$ (see [15]), we obtain

$$
\|p(B)\|=\left\|\xi_{p} \circ b\right\| \leq c\left\|\xi_{p} \circ a\right\|=c\|p(A)\|
$$

for some $c \in \mathbb{R}_{+}$. This proves the continuity of the map $\Phi: p(A) \mapsto p(B)$. Similarly we prove the continuity of $\Phi^{-1}$.

Let $f \in X, g \in X^{\star}$ and assume again that $W(B) \subset W(A)$. It follows that there exist $h \in X$ and $k \in X^{\star}$ such that

$$
\begin{aligned}
\langle C(B, \zeta) f, g\rangle & =\sum_{\alpha \geq 0} \frac{(n+|\alpha|-1) !}{(n-1) ! \alpha !}\left\langle B^{\alpha} f, g\right\rangle \bar{\zeta}^{\alpha} \\
& =\sum_{\alpha \geq 0} \frac{(n+|\alpha|-1) !}{(n-1) ! \alpha !}\left\langle A^{\alpha} h, k\right\rangle \bar{\zeta}^{\alpha}=\langle C(A, \zeta) h, k\rangle .
\end{aligned}
$$

This implies that $\left\langle B^{\alpha} f, g\right\rangle=\left\langle A^{\alpha} h, k\right\rangle$ for each $\alpha$. Consequently,

$$
\langle\Phi(p(A)) f, g\rangle=\langle p(A) h, k\rangle
$$

for each polynomial $p$. This proves that the resolvent equality implies that $\Phi^{\star}$ maps $\mathcal{P}\left(B_{1}, \ldots, B_{n}\right)_{\mathfrak{X} \times x}^{\star}$ onto $\mathcal{P}\left(A_{1}, \ldots, A_{n}\right)_{\mathfrak{X} \times x}^{\star}$. The converse implication can be proved in the same manner.

Significantly, the reproducing formula with Poisson integral can be obtained not only for polynomials or more generally for functions belonging to the ball algebra but also for functions of the form $p+\bar{q}$, where $p, q \in \mathbb{C}\left[Z_{1}, \ldots, Z_{n}\right]$.

Proposition 6. Let $A_{1}, \ldots, A_{n}$ be commuting operators on Hilbert space $\mathcal{H}$ with $\sigma_{\mathrm{T}}(A) \subset \mathbb{B}$. Then for any polynomials $p, q \in \mathbb{C}\left[Z_{1}, \ldots, Z_{n}\right]$,

$$
p(A)+q\left(A^{*}\right)=\int_{S}(p(\zeta)+q(\bar{\zeta})) P(A, \zeta) d \sigma(\zeta) .
$$


Proof. The method of proof is exactly the same as in [15].

Assume now that $\sigma_{\mathrm{T}}(A) \subset \mathbb{B}$. One can define a weak Poisson resolvent in a similar manner using the operator-valued Poisson kernel (2) instead of the Cauchy kernel, i.e. a weak Poisson resolvent is a function on $\overline{\mathbb{B}}$ of the form

$$
\psi_{f, g}(\zeta)=\langle P(A, \zeta) f, g\rangle,
$$

where $f, g \in \mathcal{H}$. The set of all weak Poisson resolvents will be denoted by $W_{P}(A)$.

Using the previous proposition, as in Theorem 5, one can prove that if $A_{1}, \ldots, A_{n}$ and $B_{1}, \ldots, B_{n}$ are commuting operators on a Hilbert space $\mathcal{H}$, then from the inclusion $W_{P}(B) \subset W_{P}(A)$ it follows that the mapping

$$
\mathcal{P}(A)+\mathcal{P}\left(A^{*}\right) \ni p(A)+q\left(A^{*}\right) \mapsto p(B)+q\left(B^{*}\right) \in \mathcal{P}(B)+\mathcal{P}\left(B^{*}\right)
$$

is linear continuous and thus can be extended to a continuous linear map on $\overline{\mathcal{P}(A)+\mathcal{P}\left(A^{*}\right)}$.

THEOREM 7. For commuting n-tuples $A$ and $B$ of operators acting on a Hilbert space $\mathcal{H}$ such that $\sigma_{\mathrm{T}}(A), \sigma_{\mathrm{T}}(B) \subset \mathbb{B}$ the equality $W_{P}(A)=W_{P}(B)$ implies $\overline{\mathcal{P}(A)+\mathcal{P}\left(A^{*}\right)} \approx \overline{\mathcal{P}(B)+\mathcal{P}\left(B^{*}\right)}$.

The proof follows the lines of the corresponding one for the Cauchy weak resolvents. The only difference is in using Proposition 6 instead of power series expansion in the proof that spatial isomorphism preserves elementary functionals.

In [13] an example was given of a pair of normal operators having the same sets of weak resolvents which are not similar on any reducing subspace. In other words, for these operators, neither the isomorphism $\Phi: \mathcal{P}(A) \rightarrow$ $\mathcal{P}(B)$ nor its inverse is of the form $X \mapsto U^{*} X U$, where $U$ is unitary.

The symbol $\mathcal{C}^{*}(A)$ will stand for the $C^{*}$-algebra generated by the $n$-tuple $A=\left(A_{1}, \ldots, A_{n}\right)$. To simplify the notation we shall sometimes write $\mathfrak{A}$ for this algebra. We will show that under the assumption $\mathcal{C}^{*}(A) \approx \mathcal{C}^{*}(B)$ (by Theorem 5 equivalent to $W\left(A, A^{*}\right)=W\left(B, B^{*}\right)$, provided the Taylor spectra of these tuples are contained in the unit ball) the map $\Phi$ has the above form on no maximal reducing subspace. We assume in this section that $\mathcal{H}$ is separable.

Denote by $E_{A}$ the joint spectral measure of the $n$-tuple $A=\left(A_{1}, \ldots, A_{n}\right)$ (see [2] for its definition and properties). The basic decomposition of a normal operator (cf. [7]) can also be carried out for commuting tuples of normal operators acting on a separable Hilbert space. Namely, it can be shown that there exist vectors $x_{0}, x_{1}, \ldots$ such that, if we define compactly supported positive measures by

$$
\mu_{k}(\Delta)=\left\langle E_{A}(\Delta) x_{k}, x_{k}\right\rangle, \quad k \in \mathbb{N},
$$


then the operators $A_{1}, \ldots, A_{n}$ are jointly unitarily equivalent to the operators $M_{i}=\oplus_{k} M_{z_{i}, k}$ given by

$$
M_{i}: \bigoplus_{k=0}^{\infty} L^{2}\left(\mu_{k}\right) \ni \oplus_{k} f_{k} \mapsto \oplus_{k} z_{i} f_{k} \in \bigoplus_{k=0}^{\infty} L^{2}\left(\mu_{k}\right)
$$

The measures $\mu_{k}$ can be chosen in such a way that $\mu_{k}$ is absolutely continuous with respect to $\mu_{k-1}$ for $k \in \mathbb{N}$. Thus there exist measurable functions $d \mu_{k} / d \mu_{0} \in L^{1}\left(\mu_{0}\right)$ such that $d \mu_{k}=\left(d \mu_{k} / d \mu_{0}\right) d \mu_{0}$. Observe that since the measures $\mu_{k}$ are positive, the functions $d \mu_{k} / d \mu_{0}$ are nonnegative a.e. for $k \in \mathbb{N}$.

Proposition 8. Let $A_{1}, \ldots, A_{n}$ be normal commuting operators on a separable Hilbert space $\mathcal{H}$ and let $y_{0} \in \mathcal{H}$. Then there exists $x \in \mathcal{H}$ such that for each $y \in \mathcal{H}$ the measure $\left\langle E_{A}(\cdot) y, y\right\rangle$ is absolutely continuous with respect to $\left\langle E_{A}(\cdot) x, x\right\rangle$ and

$$
y_{0} \in \mathcal{M}(x):=\overline{\mathfrak{A} x} .
$$

Proof. The proof can be carried out as that of Lemma 7 on p. 913 in [7].

The space $\mathcal{M}(x)$ and the vector $x$, whose existence is proved in the above proposition, are called maximal relative to $A$. If $C$ is any operator acting on $\mathcal{H}$, then we denote by $C^{(\infty)}$ its infinite inflation, i.e. $C \otimes \mathrm{id}_{\mathcal{H}}$. This operator acts on $\bigoplus_{0}^{\infty} \mathcal{H}$, the infinite orthogonal sum of copies of $\mathcal{H}$. This space will thereafter be denoted by $\mathcal{H}^{(\infty)}$. The symbol $\iota$ will stand for the inclusion of $\mathcal{H}$ onto a fixed, say the first, component of $\mathcal{H}^{(\infty)}$. If $C=\left(C_{1}, \ldots, C_{n}\right)$ is an $n$-tuple of operators acting on $\mathcal{H}$, then $C^{(\infty)}$ stands for the $n$-tuple $\left(C_{1}^{(\infty)}, \ldots, C_{n}^{(\infty)}\right)$. This convention makes clear the meaning of the symbol $p\left(C^{(\infty)}\right)$, when $p \in \mathbb{C}\left[Z_{1}, \ldots, Z_{n}\right]$.

LEMMA 9. If $x$ is maximal relative to $A$ then $\iota x$ is maximal relative to $A^{(\infty)}$.

Proof. Observe that if $E_{A}$ is a spectral measure of the $n$-tuple $A$, then $E^{(\infty)}=E \otimes \operatorname{id}_{\mathcal{H}}$ is a spectral measure of $A^{(\infty)}$. Thus, if $\left\langle E^{(\infty)}(\Delta) \iota x, \iota x\right\rangle=0$ for a Borel set $\Delta$, then for any $\oplus_{k} x_{k} \in \mathcal{H}^{(\infty)}$ we obtain

$$
\left\langle E^{(\infty)}(\Delta)\left(\oplus x_{k}\right), \oplus x_{k}\right\rangle=\sum_{k}\left\langle E(\Delta) x_{k}, x_{k}\right\rangle=0 .
$$

THEOREM 10. Assume that $A_{1}, \ldots, A_{n}$ and $B_{1}, \ldots, B_{n}$ are commuting $n$-tuples of normal operators on a Hilbert space $\mathcal{H}$. The following conditions are equivalent:

(i) $\mathrm{C}^{*}(A) \approx \mathrm{e}^{*}(B)$.

(ii) If $\mathcal{M}_{A}$ and $\mathcal{M}_{B}$ are maximal subspaces relative to $A$ and $B$, then $\left.A\right|_{\mathcal{M}_{A}}$ and $\left.B\right|_{\mathcal{M}_{B}}$ are jointly unitarily equivalent. 
(iii) There exist isometries $V, W \in \mathbf{B}\left(\mathcal{H}^{(\infty)}\right)$ such that

$$
\begin{aligned}
& p\left(A^{(\infty)}, A^{(\infty) *}\right)=V^{*} p\left(B^{(\infty)}, B^{(\infty) *}\right) V, \\
& p\left(B^{(\infty)}, B^{(\infty) *}\right)=W^{*} p\left(A^{(\infty)}, A^{(\infty) *}\right) W .
\end{aligned}
$$

Proof. (i) $\Rightarrow$ (ii). From the above-sketched model theory for normal operators and Lebesgue dominated convergence theorem it follows that for each polynomial $p$ of $2 n$ variables,

$$
\begin{aligned}
\left\langle p(A)\left(\oplus_{k} f_{k}\right), \oplus_{k} g_{k}\right\rangle & =\sum_{k} \int_{\sigma(A)} p(z, \bar{z}) f_{k}(z) \overline{g_{k}(z)} d \mu_{k}(z) \\
& =\int_{\sigma(A)} p(z, \bar{z})\left(\sum_{k} f_{k}(z) \overline{g_{k}(z)} \frac{d \mu_{k}}{d \mu_{0}}\right) d \mu_{0}(z),
\end{aligned}
$$

since one can prove as in [13] that the series

$$
\sum_{k} f_{k}(z) \overline{g_{k}(z)} \frac{d \mu_{k}}{d \mu_{0}}
$$

converges absolutely in $L_{1}\left(\mu_{0}\right)$. It is known (cf. [3], [5]) that for commuting normal operators $A_{1}, \ldots, A_{n}$ the equality $\sigma_{\mathrm{T}}(A)=\sigma_{\mathfrak{A}}(A)$ holds. Therefore, we write $\sigma(A)$ for any of the equivalent notions of spectrum.

Thus, for each subspace $\mathcal{M}_{A}$, maximal relative to $A$, we can always find vectors $f^{\prime}, g^{\prime} \in \mathcal{M}_{A}$ satisfying

$$
\left\langle p\left(A, A^{*}\right) f, g\right\rangle=\left\langle p\left(\left.A\right|_{\mathcal{M}_{A}},\left.A\right|_{\mathcal{M}_{A}} ^{*}\right) f^{\prime}, g^{\prime}\right\rangle .
$$

In other words,

$$
\mathcal{C}^{*}(A) \approx \mathcal{C}^{*}\left(\left.A\right|_{\mathcal{M}_{A}}\right)
$$

Observe that if $\mathcal{C}^{*}(A)$ and $\mathcal{C}^{*}(B)$ are spatially isomorphic, then $\sigma(A)=\sigma(B)$. Denote this set by $\sigma$.

From the previous observations and the assumption, it follows that there exist vectors $h, k \in \mathcal{M}_{B}$ such that

$$
\int_{\sigma} p(z, \bar{z}) d \mu_{0}^{A}=\int_{\sigma} p(z, \bar{z}) h(z) \overline{k(z)} d \mu_{0}^{B}
$$

for each polynomial $p$. From the Stone-Weierstrass theorem it follows that the measure $\mu_{0}^{A}$ is absolutely continuous with respect to $\mu_{0}^{B}$. Similarly we prove the inverse relation. Consequently, $\left.A\right|_{\mathcal{M}_{A}}$ and $\left.B\right|_{\mathcal{M}_{B}}$ are unitarily equivalent.

(ii) $\Rightarrow$ (i). From (8) it follows that it is enough to show that

$$
\mathrm{C}^{*}\left(\left.A\right|_{\mathcal{M}_{A}}\right) \approx \mathcal{C}^{*}\left(\left.B\right|_{\mathcal{M}_{B}}\right) \text {. }
$$

This follows easily from the assumption, since

$$
\left\langle p\left(\left.A\right|_{\mathcal{M}_{A}},\left.A\right|_{\mathcal{M}_{A}} ^{*}\right) f, g\right\rangle=\left\langle p\left(\left.B\right|_{\mathcal{M}_{B}},\left.B\right|_{\mathcal{M}_{B}} ^{*}\right) U f, U g\right\rangle .
$$


(i) $\Rightarrow$ (iii). Decompose the space $\mathcal{H}$ into reducing subspaces of the form $\overline{\mathfrak{A} f_{i}}$. Let $\mu_{i}^{A}, i \in \mathbb{N}_{0}$, be the corresponding measures. Denote by $\mathcal{A}_{i, j}, i, j \in \mathbb{N}_{0}$, the $j$ th copy of the space $\overline{\mathfrak{A} f_{i}}$ in $\mathcal{H}^{(\infty)}$. Let $\mathcal{B}_{i, j}$ be the analogous decomposition of $\mathcal{H}$ for $B$ such that $\mathcal{B}_{0, j}$ is maximal relative to $B$ for each $j \in \mathbb{N}_{0}$. Let $\phi$ be any bijection between $\mathbb{N}_{0} \times \mathbb{N}_{0}$ and $\mathbb{N}_{0}$.

From part (i) $\Rightarrow$ (ii) of the proof, it follows that $\mu_{i}^{A} \ll \mu_{0}^{A} \ll \mu_{0}^{B}, i \in \mathbb{N}$. Define an operator $V_{i, j}: \mathcal{A}_{i, j} \rightarrow \mathcal{B}_{0, \phi(i, j)}$ by

$$
V_{i, j} f=\sqrt{\frac{d \mu_{i}^{A}}{d \mu_{0}^{B}}} f .
$$

Since $d \mu_{i}^{A} / d \mu_{0}^{B}$ belongs to $L^{1}\left(\mu_{0}^{B}\right)$, the operator $V_{i, j}$ is an isometry. Consequently, the operator $V=\oplus_{i, j} V_{i, j}$ is an isometry. Straightforward computation shows that for any polynomial

$$
p\left(A^{(\infty)}, A^{(\infty) *}\right)=\Phi^{(\infty)}\left(p\left(B^{(\infty)}, B^{(\infty) *}\right)\right)=V^{*} p\left(B^{(\infty)}, B^{(\infty) *}\right) V .
$$

Similarly, we prove the existence of the isometry $W$.

(iii) $\Rightarrow$ (i). From the assumption it follows that the map $p\left(A^{(\infty)}, A^{(\infty) *}\right) \mapsto$ $p\left(B^{(\infty)}, B^{(\infty) *}\right)$ extends to a bounded isomorphism of $\mathrm{C}^{*}(A)$ and $\mathrm{C}^{*}(B)$.

Denote by $\iota: \mathcal{H} \rightarrow \mathcal{H}^{(\infty)}$ the inclusion onto the first component. By the assumption we have

$$
\left\langle p\left(A, A^{*}\right) f, g\right\rangle=\left\langle p\left(A^{(\infty)}, A^{(\infty) *}\right) \iota f, \iota g\right\rangle=\left\langle p\left(B^{(\infty)}, B^{(\infty) *}\right) V \iota f, V \iota g\right\rangle .
$$

Since for each vector $h \in \mathcal{H}$ maximal relative to $B$, $\iota h$ is maximal relative to $B^{(\infty)}$, the conclusion follows from (8).

EXAMPLE 11. In [13] an example was given of normal operators $A, B$ which satisfy the weak resolvent equality (i.e. $W(A)=W(B)$ or equivalently $\overline{\mathcal{P}(A)} \cong \overline{\mathcal{P}(B)})$ but are not unitarily equivalent on any reducing subspace. We infer that the relation $\approx$ cannot be extended from algebras $\mathcal{A}, \mathcal{B}$ of operators to $C^{*}$-algebras generated by them.

For the convenience of the reader we describe the example in detail. Let $\left\{r_{n}: n \in \mathbb{N}\right\}$ and $\left\{s_{n}: n \in \mathbb{N}\right\}$ be disjoint countable subsets of $(0,1)$, each dense in $[0,1]$. Let $\mu_{n}\left(\right.$ resp. $\left.\nu_{n}\right)$ be $2^{-n}$ times normalized Lebesgue measure on the circle with centre 0 and of radius $r_{n}$ (resp. $s_{n}$ ) for $n \in \mathbb{N}$. Put $\mu=\sum_{n \in \mathbb{N}} \mu_{n}$ and $\nu=\sum_{n \in \mathbb{N}} \nu_{n}$, so $\mu$ and $\nu$ are mutually singular. Let $A$ and $B$ be multiplication by $z$ on $L^{2}(\mu)$ and $L^{2}(\nu)$, respectively.

It is easy to show that $\mathcal{P}(A) \approx \mathcal{P}(B)$. Observe that the $C^{*}$-algebras $\mathfrak{A}$ and $\mathfrak{B}$ generated by $A$ and $B$, respectively, are spatially isomorphic. Indeed, the map which sends a function $f \in C(\overline{\mathbb{D}})$ to the operator $M_{f} \in \mathbf{B}\left(L^{2}(\mu)\right)$ of multiplication by $f$ is a continuous $*$-isomorphism. Consequently, $\mathfrak{A} \cong \mathfrak{B}$, although we have noticed that $\mathfrak{A} \not{\mathfrak{B}}$. 
Theorem 10 implies that if the weak resolvent equality holds for normal operators, then there always exist "large" subspaces on which the $n$-tuples $A$ and $B$ are unitarily equivalent. A simple example of a normal operator and its inflation shows that one cannot expect that the relevant unitaries can be extended onto the whole space. We shall refer to this remark later.

Although one cannot expect to find an isometry satisfying $\Phi(\cdot)=$ $U^{*}(\cdot) U$, acting on $\mathcal{H}$, it is always possible to write $\Phi$ as a composition of a projection $\pi: \mathcal{H}^{(\infty)} \rightarrow \mathcal{H}$ onto a fixed component, a map of the form $U^{*}(\cdot) U, U$ being an isometry in $\mathbf{B}\left(\mathcal{H}^{(\infty)}\right)$, and the inclusion $\iota$. In other words, by Theorem 10(iii), the following diagram commutes:

$$
\begin{array}{cc}
\mathcal{H} & \stackrel{\iota_{1}}{\longrightarrow} \\
\mathcal{H}^{(\infty)} \stackrel{V}{\longrightarrow} \mathcal{H}(\infty) \\
\mathcal{H} \stackrel{\pi_{1}}{\longleftarrow} \mathcal{H}^{(\infty)} \stackrel{V^{*}}{\longleftarrow} \mathcal{H}^{(\infty)}
\end{array}
$$

Sufficient conditions for the equality $P(K)=C(K)$ or

$$
\overline{\mathcal{P}(K)+\mathcal{P}(K)^{*}}=C(K)
$$

to hold, where $K \subset \mathbb{C}^{n}, n>1$, is a compact set, are much more sophisticated than in the one-dimensional case. In this connection it seems that a proper generalization of Theorem 6.3 from [8] is the following fact, which can be proved exactly like Theorem 10 .

TheOREM 12. Let $A$ and $B$ be commuting n-tuples of normal operators. Assume, additionally, that $\sigma=\sigma(A)=\sigma(B)$ and that both $\mathcal{P}(A)$ and $\mathcal{P}(B)$ are Dirichlet algebras on $\sigma$. The following conditions are equivalent:

(i) $\overline{\mathcal{P}(A)+\mathcal{P}\left(A^{*}\right)} \approx \overline{\mathcal{P}(B)+\mathcal{P}\left(B^{*}\right)}$.

(ii) If $\mathcal{M}_{A}$ and $\mathcal{M}_{B}$ are maximal subspaces relative to $A$ and $B$, then $\left.A\right|_{\mathcal{M}_{A}}$ and $\left.B\right|_{\mathcal{M}_{B}}$ are unitarily equivalent.

(iii) There exist isometries $V, W \in \mathbf{B}\left(\mathcal{H}^{(\infty)}\right)$ such that

$$
\begin{aligned}
& p\left(A^{(\infty)}, A^{(\infty) *}\right)=V^{*} p\left(B^{(\infty)}, B^{(\infty) *}\right) V, \\
& p\left(B^{(\infty)}, B^{(\infty) *}\right)=W^{*} p\left(A^{(\infty)}, A^{(\infty) *}\right) W .
\end{aligned}
$$

We have proved that if $n$-tuples of normal operators $A=\left(A_{1}, \ldots, A_{n}\right)$ and $B=\left(B_{1}, \ldots, B_{n}\right)$ have the same sets of weak resolvents, then $\mathcal{C}^{*}(A) \approx$ $\mathcal{C}^{*}(B)$. From Example 11 it follows that the existence of a spatial isomorphism between $\mathcal{C}^{*}(A)$ and $\mathcal{C}^{*}(B)$ is not sufficient for $\mathcal{C}^{*}(A) \approx \mathcal{C}^{*}(B)$. However, as we intend to show, the condition $\mathcal{C}^{*}(A) \cong \mathrm{C}^{*}(B)$ implies that the closures, in the topology of uniform convergence on compact subsets of $\mathbb{B}$, of the sets of weak resolvents are equal. 
Lemma 13. Assume that $A$ and $B$ are commuting $n$-tuples of operators on a separable Hilbert space. The algebras $\mathrm{C}^{*}(A)$ and $\mathrm{C}^{*}(B)$ are spatially isomorphic if and only if $A^{(\infty)}$ and $B^{(\infty)}$ are strongly approximately equivalent.

We will sketch the proof for convenience of the reader. However, the method is exactly the same as for one operator (cf. Theorem 42.7 and Corollary 42.8 in [4]).

Proof. If $A^{(\infty)}$ and $B^{(\infty)}$ are strongly approximately equivalent, then it is easy to show that $\mathcal{C}^{*}\left(A^{(\infty)}\right)$ and $\mathcal{C}^{*}\left(B^{(\infty)}\right)$ are spatially isomorphic. On the other hand, $\mathrm{C}^{*}\left(A^{(\infty)}\right) \cong \mathrm{C}^{*}(A)$.

Assume now that $\mathcal{C}^{*}(A)$ and $\mathcal{C}^{*}(B)$ are spatially isomorphic. Denote the spatial isomorphism by $\Phi$. Define $\Psi: \mathcal{C}^{*}(A) \rightarrow \mathcal{C}^{*}\left(B^{(\infty)}\right)$ by $\Psi(T)=\Phi(T)^{(\infty)}$. Then $\Psi$ is a $*$-homomorphism. It is enough to show that $A^{(\infty)}$ and $A^{(\infty)} \oplus$ $B^{(\infty)}$ are strongly approximately equivalent since the same reasoning may be applied to $\Psi^{-1}$.

Define a representation $\Upsilon: \mathcal{C}^{*}\left(A^{(\infty)}\right) \rightarrow \mathcal{C}^{*}\left(B^{(\infty)}\right)$ by $\Upsilon\left(T^{(\infty)}\right)=\Psi(T)$. Observe that $\Upsilon\left(\mathcal{C}^{*}\left(A^{(\infty)}\right) \cap \mathbf{K}\right)=\Upsilon(0)=0$, where $\mathbf{K}$ denotes the ideal of compact operators on $\mathcal{H}^{(\infty)}$.

Denote by id the trivial representation of $\mathcal{C}^{*}\left(A^{(\infty)}\right)$, which, by the definition, is a subalgebra of $\mathbf{B}\left(\mathcal{H}^{(\infty)}\right)$. From the Voiculescu-Arveson theorem [1], [16] it follows that id and id $\oplus \Upsilon$ are strongly approximately equivalent. This proves the lemma.

TheOREM 14. Assume that $\mathrm{C}^{*}(A)$ and $\mathrm{C}^{*}(B)$ are spatially isomorphic, where $A$ and $B$ are commuting n-tuples of normal operators satisfying the condition $\sigma(A), \sigma(B) \subset \mathbb{B}$. Then the closures of $W(A)$ and $W(B)$ in the topology of uniform convergence on compact subsets of $\mathbb{B}$ are equal.

Proof. Denote by $\Phi$ the spatial isomorphism which maps $\mathcal{C}^{*}(A)$ onto $\mathcal{C}^{*}(B)$. From Lemma 13 it follows that there exists a sequence of isometries $U_{n} \in \mathbf{B}\left(\mathcal{H}^{(\infty)}\right)$ such that

$$
\left\|\Phi^{(\infty)}\left(p\left(A^{(\infty)}, A^{(\infty) *}\right)\right)-U_{n}^{*} p\left(A^{(\infty)}, A^{(\infty) *}\right) U_{n}\right\| \rightarrow 0 .
$$

Consequently, for each $\zeta \in \mathbb{B}, \oplus_{k} f_{k}, \oplus g_{k} \in \mathcal{H}^{(\infty)}$,

$$
\left|\left\langle C\left(B^{(\infty)}, \zeta\right)\left(\oplus f_{k}\right), \oplus g_{k}\right\rangle-\left\langle U_{n}^{*} C\left(A^{(\infty)}, \zeta\right) U_{n}\left(\oplus f_{k}\right), \oplus g_{k}\right\rangle\right| \rightarrow 0 .
$$

Take $f, g \in \mathcal{H}$. From (9) it follows that for each $\zeta \in \mathbb{B}$,

$$
\langle C(B, \zeta) f, g\rangle=\lim _{n \rightarrow \infty}\left\langle C\left(A^{(\infty)}, \zeta\right) U_{n} \iota f, U_{n} \iota g\right\rangle .
$$

Set $\oplus_{k} f_{k}^{n}=U_{n} \iota f$ and $\oplus_{k} g_{k}^{n}=U_{n} \iota g$. Observe that the series

$$
\sum_{k} f_{k}^{n}(z) \overline{g_{k}^{n}(z)} \frac{d \mu_{k}}{d \mu_{1}}
$$


converges absolutely in $L_{1}\left(\mu_{1}\right)$. Therefore, as in Theorem 10,

$$
\begin{aligned}
\left\langle C\left(A^{(\infty)}, \zeta\right)\left(\oplus_{k} f_{k}^{n}\right), \oplus_{k} g_{k}^{n}\right\rangle & =\sum_{k} \int_{\sigma} C(z, \zeta) f_{k}^{n}(z) \overline{g_{k}^{n}(z)} d \mu_{k} \\
& =\int_{\sigma} C(z, \zeta)\left\{\sum_{k} f_{k}^{n}(z) \overline{g_{k}^{n}(z)} \frac{d \mu_{k}}{d \mu_{1}}\right\} d \mu_{1} \\
& =\left\langle C(A, \zeta) f_{n}^{\prime}, g_{n}^{\prime}\right\rangle,
\end{aligned}
$$

where $f_{n}^{\prime}, g_{n}^{\prime}$ are functions in $L_{2}\left(\mu_{1}\right)$ satisfying

$$
f_{n}^{\prime}(z) \overline{g_{n}^{\prime}(z)}=\sum_{k} f_{k}^{n}(z) \overline{g_{k}^{n}(z)} \frac{d \mu_{k}}{d \mu_{1}} .
$$

This yields the desired conclusion, since by the Montel theorem the sequence of holomorphic functions $\overline{\left\langle C(A, \cdot) f_{n}^{\prime}, g_{n}^{\prime}\right\rangle}$ converges uniformly on compact subsets of $\mathbb{B}$.

4. Generalizations to other operators. We continue the investigation of implications of the weak resolvent equality for other, not necessarily normal, operators acting on a Hilbert space. It is of interest what can be said about spatial isomorphism such that the dual map also preserves vector states. This fact will be denoted by $\mathcal{A} \approx_{\mathrm{s}} \mathcal{B}$. Theorem 10 and Proposition 8 show that for normal operators, $\mathcal{C}^{*}(A) \approx \mathcal{C}^{*}(B)$ implies that for each $g, h \in \mathcal{H}$ there exist subspaces $\mathcal{M}_{A} \ni g$ and $\mathcal{M}_{B} \ni h$ such that the restrictions of $A$ and $B$ to $\mathcal{M}_{A}$ and $\mathcal{M}_{B}$, respectively, are unitarily equivalent. From the next proposition it follows that this implies that $\mathcal{C}^{*}(A) \widetilde{\simeq}_{\mathrm{s}} \mathrm{C}^{*}(B)$. The symbol $\mathbb{C}\left\langle Z_{1}, \ldots, Z_{2 n}\right\rangle$ stands for the algebra of noncommutative polynomials of $2 n$ variables.

Proposition 15. Let $\mathcal{H}$ be a Hilbert space. Assume that there exist families of subspaces $\mathcal{H}_{\gamma}^{A}$ and $\mathcal{H}_{\gamma}^{B}$ such that

$$
\mathcal{H}=\bigcup_{\gamma} \mathcal{H}_{\gamma}^{A}=\bigcup_{\gamma} \mathcal{H}_{\gamma}^{B}
$$

and isometries $V_{\gamma}: \mathcal{H}_{\gamma}^{A} \rightarrow \mathcal{H}_{\gamma}^{B}$ and $W_{\gamma}: \mathcal{H}_{\gamma}^{B} \rightarrow \mathcal{H}_{\gamma}^{A}$ such that

$$
\left.p\left(A, A^{*}\right)\right|_{\mathcal{H}_{\gamma}^{A}}=V_{\gamma}^{*} p\left(B, B^{*}\right) V_{\gamma} \quad \text { and }\left.\quad p\left(B, B^{*}\right)\right|_{\mathcal{H}_{\gamma}^{B}}=W_{\gamma}^{*} p\left(A, A^{*}\right) W_{\gamma}
$$

for all $p \in \mathbb{C}\left\langle Z_{1}, \ldots, Z_{2 n}\right\rangle$. Then $\mathcal{C}^{*}(A) \approx_{\mathrm{S}} \mathrm{C}^{*}(B)$.

Proof. Let $f \in \mathcal{H}$. Then there exists $\gamma$ such that $f \in \mathcal{H}_{\gamma}^{A}$. Thus

$$
\left\langle p\left(A, A^{*}\right) f, f\right\rangle=\left\langle V_{\gamma}^{*} p\left(B, B^{*}\right) V_{\gamma} f, f\right\rangle=\left\langle p\left(B, B^{*}\right) V_{\gamma} f, V_{\gamma} f\right\rangle
$$

for each polynomial $p \in \mathbb{C}\left\langle Z_{1}, \ldots, Z_{2 n}\right\rangle$. Similarly we prove the reverse inclusion. 
Let $S$ and $T$ be $n$-tuples of operators, not necessarily normal, acting on a Hilbert space $\mathcal{H}$. The symbol $\mathcal{W}^{*}(S)$ stands for the smallest von Neumann algebra generated by $S$. We write $X^{\prime}$ to denote the commutant of the set $X \subset \mathbf{B}(\mathcal{H})$ in $\mathbf{B}(\mathcal{H})$.

Proposition 16. Assume that $\mathrm{C}^{*}(A) \approx_{\mathrm{S}} \mathrm{e}^{*}(B)$. Then the map

$$
\Phi: p\left(A, A^{*}\right) \mapsto p\left(B, B^{*}\right),
$$

where $p \in \mathbb{C}\left\langle Z_{1}, \ldots, Z_{2 n}\right\rangle$, extends to a continuous $*$-homomorphism between the SOT-closures of $\mathrm{C}^{*}(A)$ and $\mathrm{C}^{*}(B)$, when both these spaces are equipped with either the SOT, WOT or norm topology. Furthermore, the dual of $\Phi$ maps the set of vector states of $\mathcal{W}^{*}(B)$ onto the set of vector states of $\mathcal{W}^{*}(A)$, i.e. $\mathcal{W}^{*}(A) \approx_{\mathrm{s}} \mathcal{W}^{*}(B)$.

Proof. For $T=(\mathrm{SOT}) \lim T_{\alpha}$ with $T_{\alpha} \in \mathrm{C}^{*}(A)$ and $\sup _{\alpha}\left\|T_{\alpha}\right\|<\infty$ one defines $\Phi(T)=(\mathrm{SOT}) \lim _{\alpha} \Phi\left(T_{\alpha}\right)$ (abusing the notation we use the same symbol for $\Phi$ and its extension). The limit exists, since, by assumption,

$$
\text { for all } f \in \mathcal{H} \text { there exists } g \in \mathcal{H} \text { satisfying }\langle T g, g\rangle=\langle\Phi(T) f, f\rangle
$$

for all $T \in \mathcal{C}^{*}(A)$. Consequently, condition (10) must also hold for $T \in$ $\mathcal{W}^{*}(A)$. This implies that $\Phi$ extends to a SOT-SOT and WOT-WOT continuous map between $\mathcal{W}^{*}(A)$ and $\mathcal{W}^{*}(B)$.

It is also easy to observe that $\Phi$ and consequently its extension is multiplicative. Indeed, multiplication is separately SOT continuous. Therefore, $\Phi(S T)=\Phi(S) \Phi(T)$ for $S \in \mathcal{P}\left(A, A^{*}\right)$ and $T \in{\overline{\mathcal{P}}\left(B, B^{*}\right)}^{\text {SOT }}$. Repeating the argument shows that $\Phi(S T)=\Phi(S) \Phi(T)$ for $S \in \overline{\mathcal{P}\left(A, A^{*}\right)}$ SOT and $T \in{\overline{\mathcal{P}\left(B, B^{*}\right)}}^{\mathrm{SOT}}$.

The fact that $\Phi: \mathcal{W}^{*}(A) \rightarrow \mathcal{W}^{*}(B)$ is a $*$-homomorphism follows from norm continuity of $\Phi$ and the fact that $\left.\Phi\right|_{\mathcal{P}\left(A, A^{*}\right)}$ is a $*$-homomorphism.

If $\mathcal{A}$ is a subspace of $\mathbf{B}(\mathcal{H})$, then an $\mathcal{A}$-cyclic subspace of $\mathcal{H}$ is a space of the form $\overline{\mathcal{A} h}$, where $h \in \mathcal{H}$. The orthogonal projection on this space will be called a cyclic projection and denoted by $[\mathcal{A} h]$. We will use the symbol $[\mathcal{A} h]$ for the projection or its image. The next lemma is well known.

Lemma 17. Let $\mathfrak{A}$ be a $C^{*}$-subalgebra of $\mathbf{B}(\mathcal{H})$. There exists a family of unit vectors $h_{\gamma}$ such that

$$
\sum_{\gamma}\left[\mathfrak{A} h_{\gamma}\right]=I
$$

One of the vectors $h_{\gamma}$ can be chosen completely arbitrary.

Let $S$ and $T$ be $n$-tuples of operators acting on a Hilbert space. Observe that neither $S$ nor $T$ has to be commutative. 
Theorem 18. If $\mathcal{C}^{*}(S) \approx_{\mathrm{s}} \mathrm{C}^{*}(T)$, then there exists a partial isometry $V$ such that

$$
p\left(S, S^{*}\right)=V^{*} p\left(T, T^{*}\right) V
$$

on its initial space. The initial or final space of $V$ can be chosen to contain a fixed arbitrary vector. If there exists an $m$-tuple $A$ belonging to the centre of $\mathcal{W}^{*}(S)$ and vectors $x_{\gamma}$ such that

$$
\sum_{\gamma}\left[\mathfrak{A}^{\prime} x_{\gamma}\right]=I
$$

and $\mathcal{C}^{*}(S) x_{\gamma}$ is dense in $\left[\mathfrak{A}^{\prime} x_{\gamma}\right]$, then there exists an isometry $V$ satisfying (11). The symbol $\mathfrak{A}$ stands for $\mathfrak{C}^{*}(A)$.

The most important step in the proof of this theorem is the following:

Proposition 19. Let $A=\left(A_{1}, \ldots, A_{n}\right)$ and $B=\left(B_{1}, \ldots, B_{n}\right)$ be $n$ tuples of operators. Denote the $C^{*}$-algebras generated by these tuples by $\mathfrak{A}$ and $\mathfrak{B}$, respectively. Assume that

$$
\sum_{\gamma}\left[\mathfrak{A}^{\prime} x_{\gamma}\right]=I
$$

for some vectors $x_{\gamma}$. If $\mathrm{C}^{*}(A) \approx_{\mathrm{S}} \mathrm{e}^{*}(B)$ then there exist vectors $y_{\gamma}$ such that

$$
\begin{gathered}
\sum_{\gamma}\left[\mathfrak{B}^{\prime} y_{\gamma}\right]=I, \\
\left\|p\left(A, A^{*}\right) x_{\gamma}\right\|=\left\|p\left(B, B^{*}\right) y_{\gamma}\right\| .
\end{gathered}
$$

In other words, if $\mathcal{C}^{*}(A) \approx_{\mathrm{S}} \mathcal{C}^{*}(B)$ then for each decomposition of the Hilbert space $\mathcal{H}$ into $\mathfrak{A}^{\prime}$-cyclic subspaces, there exists a corresponding decomposition into $\mathfrak{B}^{\prime}$-cyclic subspaces.

In the proof of Proposition 19 we will repeatedly make use of the following well known facts.

Lemma 20. Let $C$ be a $C^{*}$-subalgebra of $\mathbf{B}(\mathcal{H})$. Then

(i) The cyclic projections for $C$ commute with $C$.

(ii) Conversely, if $Q$ is a projection in the commutant $C^{\prime}$ and $x \in R(Q)$ then $Q \geq[C x]$.

Proof of Proposition 19. From the assumption $\mathfrak{A}=\mathfrak{C}^{*}(A) \widetilde{\simeq}_{\mathrm{s}} \mathcal{C}^{*}(B)=\mathfrak{B}$ it follows that $\Phi$ is a $*$-isomorphism between $\overline{\mathfrak{A}}^{\mathrm{SOT}}$ and $\overline{\mathfrak{B}}^{\mathrm{SOT}}$. From the von Neumann double commutant theorem we know that these closures are $\mathfrak{A}^{\prime \prime}$ and $\mathfrak{B}^{\prime \prime}$, respectively.

Since $\Phi$ is a $*$-isomorphism and the projection $\left[\mathfrak{A}^{\prime} x_{\gamma}\right]$ belongs to the double commutant of $\mathfrak{A}$ for each $\gamma$, it follows that $\Phi\left(\left[\mathfrak{A}^{\prime} x_{\gamma}\right]\right)$ is a projection. Furthermore, $\left\{\left[\mathfrak{A}^{\prime} x_{\gamma}\right]\right\}$ is a family of mutually orthogonal projections and 
thus its image under $\Phi$ has the same property. From the SOT continuity of $\Phi$ we also have the equality

$$
\sum_{\gamma} \Phi\left(\left[\mathfrak{A}^{\prime} x_{\gamma}\right]\right)=I .
$$

By Proposition 16 there exist vectors $y_{\gamma}$ such that

$$
\left\|F x_{\gamma}\right\|=\left\|\Phi(F) y_{\gamma}\right\|
$$

for any $F \in \mathcal{W}^{*}(A)$. Thus we may write

$$
\left\|y_{\gamma}\right\|=\left\|x_{\gamma}\right\|=\left\|\left[\mathfrak{A}^{\prime} x_{\gamma}\right] x_{\gamma}\right\|=\left\|\Phi\left(\left[\mathfrak{A}^{\prime} x_{\gamma}\right]\right) y_{\gamma}\right\| .
$$

It follows that $y_{\gamma}$ belongs to the range of $\Phi\left(\left[\mathfrak{A}^{\prime}\right] x_{\gamma}\right)$. Since the projections $\Phi\left(\left[\mathfrak{A}^{\prime} x_{\gamma}\right]\right)$ belong to the double commutant of $\mathfrak{B}$ we have $\Phi\left(\left[\mathfrak{A}^{\prime} x_{\gamma}\right]\right) \geq\left[\mathfrak{B}^{\prime} y_{\gamma}\right]$. Thus the projections $\left[\mathfrak{B}^{\prime} y_{\gamma}\right]$ are mutually orthogonal.

We will show that

$$
\left[\mathfrak{B}^{\prime} y_{\gamma}\right]=\Phi\left[\mathfrak{A}^{\prime} x_{\gamma}\right]
$$

Since $\left[\mathfrak{B}^{\prime} y_{\gamma}\right] \in \mathfrak{B}^{\prime \prime}$ we may define a family of mutually orthogonal projections by $Q_{\gamma}=\Phi^{-1}\left[\mathfrak{B}^{\prime} y_{\gamma}\right]$. Observe that from the continuity of the $*$-isomorphism $\Phi^{-1}$ it follows that

$$
\left\|Q_{\gamma} x_{\gamma}\right\|=\left\|\left[\mathfrak{B}^{\prime} y_{\gamma}\right] y_{\gamma}\right\|=\left\|y_{\gamma}\right\|=\left\|x_{\gamma}\right\| .
$$

In other words, $x_{\gamma}$ belongs to the range of $Q_{\gamma}$ for each $\gamma$. Thus, $\left[\mathfrak{A}^{\prime} x_{\gamma}\right] \leq Q_{\gamma}$. Since the $*$-isomorphism $\Phi$ preserves $\leq$, we get $\Phi\left(\left[\mathfrak{A}^{\prime} x_{\gamma}\right]\right) \leq \Phi\left(Q_{\gamma}\right)=\left[\mathfrak{B}^{\prime} y_{\gamma}\right]$, which completes the proof.

Proof of Theorem 18. It follows from the assumption that for each $f \in \mathcal{H}$ there exists $h \in \mathcal{H}$ such that for any noncommutative polynomial $p$,

$$
\left\langle p\left(S, S^{*}\right) f, f\right\rangle=\left\langle p\left(T, T^{*}\right) g, g\right\rangle \text {. }
$$

Thus the first part is trivial. Let $V: \overline{\mathrm{C}^{*}(S) f} \rightarrow \overline{\mathrm{e}^{*}(T) g}$ be a linear operator which continuously extends the map $p\left(S, S^{*}\right) f \mapsto p\left(T, T^{*}\right) g$.

Straightforward computations yield

$$
p\left(S, S^{*}\right)=V^{*} p\left(T, T^{*}\right) V
$$

on $\mathcal{C}^{*}(S) f$. It remains to extend $V$ onto the whole space $\mathcal{H}$ composing with the projection on the closure of $\mathrm{e}^{*}(S) f$.

To prove the second part, denote by $B$ the image of $A$ under $\Phi$. Observe that

$$
\mathfrak{A}=\mathfrak{C}^{*}(A) \approx_{\mathrm{s}} \mathrm{C}^{*}(B)=\mathfrak{B},
$$

since $\mathrm{C}^{*}(S) \approx_{\mathrm{s}} \mathrm{e}^{*}(T)$. By assumption there exist $x_{\gamma}$ such that

$$
\sum_{\gamma}\left[\mathfrak{A}^{\prime} x_{\gamma}\right]=I .
$$


One can choose $y_{\gamma}$ satisfying

$$
\sum_{\gamma}\left[\mathfrak{B}^{\prime} y_{\gamma}\right]=I \quad \text { and } \quad\left\|p\left(S, S^{*}\right) x_{\gamma}\right\|=\left\|p\left(T, T^{*}\right) y_{\gamma}\right\|
$$

for $p \in \mathbb{C}\left\langle Z_{1}, \ldots, Z_{2 n}\right\rangle$. This can be done as in the proof of Proposition 19 , but for the $*$-isomorphism which implements the equivalence relation $\mathcal{W}^{*}(S) \approx_{\mathrm{s}} \mathcal{W}^{*}(T)$. It is now straightforward to complete the proof.

Define $\mathfrak{A}=\mathfrak{C}^{*}(A)$ and $\mathfrak{B}=\mathfrak{C}^{*}(B)$. To complete the investigation of the consequences of the weak resolvent equality one would also need to understand when $\mathfrak{A} \approx \mathfrak{B}$ implies that $\mathfrak{A} \approx_{\mathrm{S}} \mathfrak{B}$.

Theorem 21. Let $\mathfrak{A}, \mathfrak{B}$ be $\mathcal{C}^{*}$-algebras generated by $n$-tuples $A$ and $B$, respectively. Assume that both $\mathfrak{A}$ and $\mathfrak{B}$ are irreducible. If $\mathfrak{A} \approx \mathfrak{B}$, then $\mathfrak{A} \approx_{\mathrm{s}} \mathfrak{B}$. Consequently, there exists a unitary operator $U$ such that

$$
p\left(S, S^{*}\right)=U^{*} p\left(T, T^{*}\right) U .
$$

Proof. We have to show that for each $f \in \mathcal{H}$ there exists $k \in \mathcal{H}$ such that

$$
\langle a f, f\rangle=\langle\Phi(a) k, k\rangle
$$

for each $a \in \mathfrak{A}$. The converse implication could be proved similarly. By assumption, for each $f \in \mathcal{H}$, which obviously may be assumed not to be 0 , there exist $g, h \in \mathcal{H}$ such $\langle a f, f\rangle=\langle\Phi(a) g, h\rangle$ for every $a \in \mathfrak{A}$. Take any $b \in \mathfrak{B}$. Since $\Phi$ is an isomorphism, there exists $a \in \mathfrak{A}$ such that $b=\Phi(a)$. Then

$$
\left\langle b^{*} b g, h\right\rangle=\left\langle\Phi\left(a^{*} a\right) g, h\right\rangle=\left\langle a^{*} a f, f\right\rangle=\|a f\|^{2} \geq 0 .
$$

If $g$ and $h$ are linearly dependent, say $h=\alpha g, \alpha \in \mathbb{C}$, then from (15) it follows easily that $\alpha \in \mathbb{R}_{+}$. Consequently, defining $k=\sqrt{\alpha} g$ we obtain (14).

Assume now that $g$ and $h$ are linearly independent, hence each is nonzero. From the Kadison transitivity theorem it follows that there exists $c \in \mathfrak{B}$ such that $c g=g$ and $c h=-g$. Thus

$$
0 \leq\left\langle c^{*} c g, h\right\rangle=\langle c g, c h\rangle=-\|g\|^{2} .
$$

Consequently, $g$ and $h$ have to be linearly dependent. This proves the first part of the theorem.

Since the dual map of $\Phi$ preserves vector states, the proof of the second part is exactly the same as the proof of Theorem 18. Just notice that if $S$ is irreducible, then each non-zero vector is cyclic. Consequently, the partial isometry can be extended onto the whole space and has dense image.

\section{References}

[1] W. Arveson, Notes on extensions of $C^{*}$-algebras, Duke Math. J. 44 (1977), 329-354.

[2] M. Sh. Birman and M. Z. Solomjak, Spectral Theory of Selfadjoint Operators in Hilbert Space, Math. Appl. (Soviet Ser.), Reidel, Dordrecht, 1987. 
[3] A. Brown, On a class of operators, Proc. Amer. Math. Soc. 4 (1953), 723-728.

[4] J. B. Conway, A Course in Operator Theory, Grad. Stud. in Math. 21, Amer. Math. Soc., Providence, 2000.

[5] R. E. Curto, Applications of several complex variables to multiparameter spectral theory, in: Surveys of Some Recent Results in Operator Theory, Vol. II, Pitman Res. Notes in Math. 192, Longman, Harlow, 1988, 25-90.

[6] N. Dunford, Spectral operators, Pacific J. Math. 4 (1954), 321-354.

[7] N. Dunford and J. T. Schwartz, Linear Operators, Part II: Spectral Theory, Interscience, 1963.

[8] C. K. Fong, E. A. Nordgren, H. Radjavi, and P. Rosenthal, Weak resolvents of linear operators II, Indiana Univ. Math. J. 39 (1990), 67-83.

[9] J. Janas, On a theorem of Lebow and Mlak for several commuting operators, Studia Math. 76 (1983), 249-253.

[10] S. G. Krantz, Function Theory of Several Complex Variables, Wiley, New York, 1982.

[11] W. Mlak, On a theorem of Lebow, Ann. Polon. Math. 35 (1977), 107-109.

[12] N. K. Nikol'skiı̌, A Tauberian theorem for the spectral radius, Sibirsk. Mat. Zh. 18 (1977), 1367-1372 (in Russian).

[13] E. Nordgren, H. Radjavi, and P. Rosenthal, Weak resolvents of linear operators, Indiana Univ. Math. J. 36 (1987), 913-934.

[14] F.-H. Vasilescu, Residually decomposable operators in Banach spaces, Tôhoku Math. J. 21 (1969), 509-522.

[15] —, An operator-valued Poisson kernel, J. Funct. Anal. 110 (1992), 42-72.

[16] D. Voiculescu, A non-commutative Weyl-von Neumann theorem, Rev. Roumaine Math. Pures Appl. 21 (1976), 97-113.

Faculty of Mathematics and Computer Science

Adam Mickiewicz University

Umultowska 87

61-614 Poznań, Poland

E-mail: mjk@amu.edu.pl

Received February 27, 2003

Revised version October 25, 2005 